\title{
A Measure of Comovement for Economic Variables: Theory and Empirics
}

\author{
Christophe Croux * Mario Forni ${ }^{\dagger} \quad$ Lucrezia Reichlin ${ }^{\ddagger}$
}

\begin{abstract}
Keywords: Business cycle, Sectoral comovements, coherence, geography.

This paper proposes a measure of dynamic comovement between (possibly many) time series and names it cohesion. The measure is defined in the frequency domain and is appropriate for processes which are costationary, possibly after suitable transformations. In the bivariate case, the measure reduces to dynamic correlation and is related, but not equal, to the well known quantities coherence and coherency. Dynamic correlation on a frequency band equals (static) correlation of band-pass filtered series. Moreover, long run correlation and cohesion relate in a simple way to cointegration.

Cohesion is useful to study problems of business cycle synchronization, to investigate short-run and long-run dynamic properties of multiple time series, to identify dynamic clusters. We use state income data for the US and GDP data for European nations to provide an empirical illustration focused on the geographical aspects of business cycle fluctuations.
\end{abstract}

JEL Classification: E3, C1

Correspondence Address: Christophe Croux, ECARES, 50 ave. F.D. Roosevelt, Bruxelles 1050. e-mail: ccroux@ulb.ac.be.

\footnotetext{
${ }^{*}$ Université Libre de Bruxelles, ECARES

${ }^{\dagger}$ University of Modena and CEPR

‡Université Libre de Bruxelles, ECARES and CEPR.
} 


\section{Introduction ${ }^{1}$}

There are few empirical relations which have the status of "stylized facts" in economics. One of them is that macroeconomic aggregates comove. This observation has been the source of speculation of economic theory since its birth. In modern theories of the business cycle people have asked whether comovements can be explained by large aggregate shocks, monetary or real, or whether an explanation should be found in non-linear propagation mechanisms. Every macroeconomic textbook starts by a statement on comovements between aggregates. However, paradoxically, this is one of the facts that is least well documented and on what there is more confusion of meaning and terminology. "Comovement" is a lose term, possibly describing different phenomena and, consequently, with many different interpretations. What are really the stylized facts and what should macroeconomics be trying to explain? Appropriate measures of comovement between time series processes should be developed to provide a meaningful answer to this question. The informal discussion on comovements usually refers to something close to a notion of correlation. However, the traditional way with which the time series literature has dealt with measurement of comovements is based on a notion of rank reduction (see Ahn and Reinsel, 1988) which has a different meaning. In this category belongs the idea of cointegration (Engle and Granger, 1987): two processes are cointegrated if the spectral density at frequency zero has rank one, codependence (Gourieroux and Peaucelle, 1992), which refers to linear combinations of correlated processes which are of lower autoregressive order than others, common features (Engle and Kozicki, 1993), i.e. linear combinations which are unpredictable with respect to past information and common cycles (Vahid and Engle 1993) which are defined as common features in first differences for processes which are cointegrated. This class of concepts presents several problems. First, high cross-correlation neither implies nor is implied by cointegration, common cycles or common features (Quah, 1993 and Forni and Reichlin, 1999). Second, these measures are binary. For example, two processes are either cointegrated or not, but we can't establish different degrees of association. Finally, in order to test for rank reduction, we need to estimate the parameters of a VAR, which may be problematic

\footnotetext{
${ }^{1}$ This research has been supported by an A.R.C. contract of the Communauté française de Belgique and by the European Commission under the Training and Mobility of Researchers Programme (Contract No ERBFMRXCT98-0213)
} 
when the number of time series is large. For all these reasons, while the notion of rank reduction is certainly interesting to characterize some aspects of the dynamic properties of multivariate time series, it is not the appropriate one for the study of comovements.

But what is an appropriate measure of comovement? In this paper we show that existing textbook quantities like coherence and coherency, which are widely used in time series literature, are not appropriate as comovement indexes. We propose a related but different measure, dynamic correlation, which arise quite naturally from basic frequency domain notions. Dynamic correlation can be decomposed by frequency and frequency band and can then be used to study business cycle as well as long-run questions. Dynamic correlation between two processes over a band turns out to be identical to static correlation of the same processes, after suitable pre-filtering. Moreover, long-run dynamic correlation is related in a simple way to stochastic cointegration.

We use our notion of dynamic correlation to construct a multivariate index of comovement, which we name cohesion. The latter provides a summary measure of the degree of comovement within a group of variables or between two groups of variables and can be used, for instance, as a metric to construct dynamic clusters.

To illustrate our proposed measure and to provide further motivation, we estimate cohesion of output data in US states and European nations and study the following questions. Are output fluctuations within Europe more correlated than output fluctuations within the US and are results the same for business cycle frequencies and the long run? Are states or countries which comove more strongly closely located from a geographical point of view? Does border matters for output synchronization and at what frequency range?

\section{Dynamic correlation}

\subsection{The definition and the basic motivation}

Consider two zero-mean real stochastic processes $x$ and $y$. Let $S_{x}(\lambda)$ and $S_{y}(\lambda),-\pi \leq \lambda<\pi$, be the spectral density functions of $x$ and $y$ and $C_{x y}(\lambda)$ be the co-spectrum. The measure we propose, dynamic correlation, is

$$
\rho_{x y}(\lambda)=\frac{C_{x y}(\lambda)}{\sqrt{S_{x}(\lambda) S_{y}(\lambda)}},
$$


To motivate this measure we introduce the spectral decomposition of the processes $x_{t}$ and $y_{t}$, i.e.

$$
x_{t}=\int_{-\pi}^{\pi} e^{i \lambda t} d Z_{x}(\lambda) \quad y_{t}=\int_{-\pi}^{\pi} e^{i \lambda t} d Z_{y}(\lambda)
$$

where $d Z_{x}(\lambda)$ and $d Z_{y}(\lambda)$ are (complex) orthogonal increment processes (see e.g. Brockwell and Davis, Chap. 4). Expression 2 says that $x_{t}$ and $y_{t}$ can be expressed as 'infinite sums' of waves of different frequencies, each having a random amplitude. As is well known, the spectral and cross-spectral density functions of $x_{t}$ and $y_{t}$ are related to the above representation in the following way:

$$
\begin{aligned}
S_{x}(\lambda) & =\operatorname{var}\left(e^{i \lambda t} d Z_{x}(\lambda)\right)=\operatorname{var}\left(d Z_{x}(\lambda)\right) \\
S_{y}(\lambda) & =\operatorname{var}\left(d Z_{x}(\lambda)\right) \\
S_{x y}(\lambda) & =\operatorname{cov}\left(d Z_{x}(\lambda), d Z_{y}(\lambda)\right) \\
S_{y x}(\lambda) & =\operatorname{cov}\left(d Z_{y}(\lambda), d Z_{x}(\lambda)\right) .
\end{aligned}
$$

While the waves appearing in representation (2) are complex, it is easily seen that, if $x_{t}$ is real, then $d Z_{x}(\lambda)=\overline{d Z_{x}(-\lambda)}$, so that

$$
e^{i \lambda t} d Z_{x}(\lambda)+e^{-i \lambda t} d Z_{x}(-\lambda)=2 \cos (\lambda t) d U_{x}(\lambda)-2 \sin (\lambda t) d V_{x}(\lambda)
$$

where $d U_{x}$ and $d V_{x}$ denote, respectively, the real and the imaginary part of $d Z_{x}$. Hence we have the alternative representation

$$
x_{t}=2 \int_{0}^{\pi} \cos (\lambda t) d U_{x}(\lambda)-2 \int_{0}^{\pi} \sin (\lambda t) d V_{x}(\lambda),
$$

where the component at frequency $\lambda$ is $\cos (\lambda t) d U_{x}(\lambda)-\sin (\lambda t) d V_{x}(\lambda)$, which is real. Similarly, the real wave decomposition of $y_{t}$ is

$$
y_{t}=2 \int_{0}^{\pi} \cos (\lambda t) d U_{y}(\lambda)-2 \int_{0}^{\pi} \sin (\lambda t) d V_{y}(\lambda) .
$$

As is easily obtained from (3) and (4), our proposed measure, $\rho_{x y}(\lambda)$, is nothing else than the correlation coefficient between the real waves of frequency $\lambda$ appearing in the above representation, i.e. $\cos (\lambda t) d U_{y}(\lambda)-\sin (\lambda t) d V_{y}(\lambda)$ and $\cos (\lambda t) d U_{x}(\lambda)-\sin (\lambda t) d V_{x}(\lambda)$. This is the reason why the measure is defined only for non-negative frequencies, on the interval $[0, \pi)$. Clearly, dynamic correlation can vary between -1 and 1 . 


\subsection{The relation with "coherency" and "coherence"}

Dynamic correlation is strictly related to both coherency and coherence - two well known indeces in time series literature. Coherency is defined as

$$
\mathcal{C}_{x y}(\lambda)=\frac{S_{x y}(\lambda)}{\sqrt{S_{x}(\lambda) S_{y}(\lambda)}}=\frac{C_{x y}(\lambda)+i Q_{x y}(\lambda)}{\sqrt{S_{x}(\lambda) S_{y}(\lambda)}},
$$

where $Q_{x y}(\lambda)$ the quadrature spectrum. Hence coherency is complex in general and is not symmetric, i.e. $\mathcal{C}_{x y}(\lambda)$ and $\mathcal{C}_{y x}(\lambda)$ are not equal, but conjugate. Dynamic correlation $\rho_{x y}(\lambda)$ is the real part of coherency and can also be obtained by averaging coherencies at frequency $\lambda$ and $-\lambda$, which also are conjugate:

$$
\rho_{x y}(\lambda)=\frac{\mathcal{C}_{x y}(\lambda)+\mathcal{C}_{x y}(-\lambda)}{2} .
$$

By using (3) the reader can easily verify that $\mathcal{C}_{x y}(\lambda)$ is the correlation coefficient of $d Z_{x}(\lambda)$ and $d Z_{y}(\lambda)$. Hence the interpretation of coherency is quite similar to that of dynamic correlation, the basic difference being that the former is related to the complex representation (2) rather than the real wave decomposition (5). Put another way, we simply collect together frequencies $\lambda$ and $-\lambda$ so that the imaginary parts cancel out. This is perfectly consistent with our aim, since the waves of frequencies $\lambda$ and $-\lambda$ have the same periodicity.

Regarding coherence - or squared-coherency, as is sometimes called - it is defined as the squared modulus of coherency, that is

$$
H_{x y}(\lambda)=\frac{C_{x y}(\lambda)^{2}+Q_{x y}(\lambda)^{2}}{S_{x}(\lambda) S_{y}(\lambda)}=\frac{\left|S_{x y}(\lambda)\right|^{2}}{S_{x}(\lambda) S_{y}(\lambda)} .
$$

Therefore coherence is real and symmetric. However, it does not measure correlation at different frequencies, because it disregards the phase differences between variables. This can be easily seen by observing that $H_{x y}(\lambda)$ is invariant with respect to shifting the processes over time, i.e. the coherence between $x_{t}$ and $y_{t-k}$ is the same as that of $x_{t}$ and $y_{t}$. This, besides being an immediate consequence of the definition, is consistent with the well-known interpretation of coherence as the $R^{2}$ from the regression of $y_{t}$ on the past, present and future of $x_{t}$ : clearly, in this regression, whether the contemporaneous regressor is $x_{t}$ or $x_{t-k}$ cannot make any difference.

A different way to see the same point is provided by the polar representation of the cross-spectrum:

$$
S_{x y}(\lambda)=R_{x y}(\lambda) e^{i \theta(\lambda)}
$$




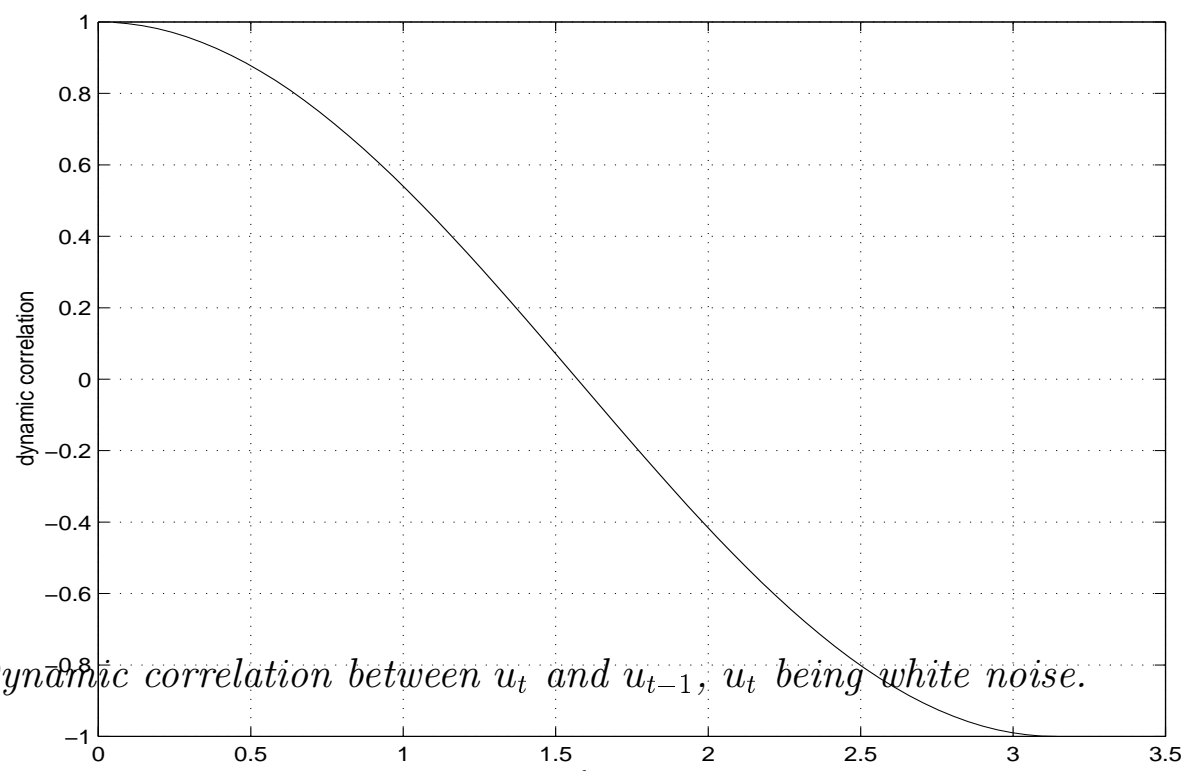

where $R_{x y}(\lambda)$ is the gain while $\theta(\lambda)$ is the phase. Breang taking the moduli on both sides we see that $\mid S_{x y}\left(\left.\lambda\right|^{2}=R_{x y}(\lambda)^{2}\right.$, so that in $H_{x y}(\lambda)$ the information contained in the phase is lost.

An illustrative example of what this entails, is provided by considering the two unit variance white noise processes $u_{t}$ and $u_{t-1}$. Their coherence is equal to one at all frequencies, despite the fact the contemporaneous correlation of the two processes is zero. By contrast, as shown in Figure 1, dynamic correlation ranges from 1, at frequency zero, to -1 at frequency $\pi$. The contemporaneous correlation zero is obtained by averaging over frequencies. We have perfect positive correlation only in the long run, which is quite intuitive. The intuition for the perfect negative correlation at frequency $\pi$, i.e. at periodicity 2 , is also simple: the waves of periodicity two are perfectly in opposition with themselves lagged one period.

The relation between dynamic correlation and static correlation is particularly simple in the case of $u_{t}$ and $u_{t-1}$ : the latter is the simple mean of the former over the interval $[0, \pi)$. This is due to the fact that $u_{t}$ has spectrum 1 at all frequencies. In the general case, this simple relation does not hold: in order to obtain the static correlation, a more complex function of the correlations at different frequencies is needed. More generally, the relation between $\rho_{x y}(\lambda)$ and $\operatorname{corr}\left(x_{t}, y_{t-k}\right)$ for different $k$ is not simple. These points are better seen after introducing the concept of dynamic correlation for a frequency band, which we do in the following subsection. 


\subsection{Dynamic correlation on a frequency band}

Let us begin by considering the processes obtained by summing the waves of $x_{t}$ and $y_{t}$ within a given frequency interval. Set $\Lambda_{+}=\left[\lambda_{1}, \lambda_{2}\right), 0 \leq \lambda_{1}<\lambda_{2} \leq \pi, \Lambda_{-}=\left[-\lambda_{2},-\lambda_{1}\right)$, $\Lambda=\Lambda_{+} \cup \Lambda_{-}$and

$$
\begin{aligned}
& x_{\Lambda t}=\int_{\Lambda} e^{i \lambda t} d Z_{x}(\lambda)=2 \int_{\Lambda_{+}} \cos (\lambda t) d U_{x}(\lambda)-2 \int_{\Lambda_{+}} \sin (\lambda t) d V_{x}(\lambda) \\
& y_{\Lambda t}=\int_{\Lambda} e^{i \lambda t} d Z_{y}(\lambda)=2 \int_{\Lambda_{+}} \cos (\lambda t) d U_{y}(\lambda)-2 \int_{\Lambda_{+}} \sin (\lambda t) d V_{y}(\lambda) .
\end{aligned}
$$

As is well known, we have

$$
\begin{aligned}
\operatorname{var}\left(x_{\Lambda t}\right) & =\int_{\Lambda} S_{x}(\lambda) d \lambda=2 \int_{\Lambda_{+}} S_{x}(\lambda) d \lambda \\
\operatorname{var}\left(y_{\Lambda t}\right) & =\int_{\Lambda} S_{y}(\lambda) d \lambda=2 \int_{\Lambda_{+}} S_{y}(\lambda) d \lambda \\
\operatorname{cov}\left(x_{\Lambda t}, y_{\Lambda t}\right) & =\int_{\Lambda} S_{x y}(\lambda) d \lambda=2 \int_{\Lambda_{+}} C_{x y}(\lambda) d \lambda .
\end{aligned}
$$

We can then define the correlation of $x_{t}$ and $y_{t}$ within the frequency band $\Lambda_{+}$as the (static) correlation coefficient of $x_{\Lambda t}$ and $y_{\Lambda t}$, i.e.

$$
\rho_{x y}\left(\Lambda_{+}\right)=\frac{\int_{\Lambda_{+}} C_{x y}(\lambda) d \lambda}{\sqrt{\int_{\Lambda_{+}} S_{x}(\lambda) d \lambda \int_{\Lambda_{+}} S_{y}(\lambda) d \lambda}}=\frac{\int_{\Lambda_{+}} \rho_{x y}(\lambda) \sqrt{S_{x}(\lambda) S_{y}(\lambda)} d \lambda}{\sqrt{\int_{\Lambda_{+}} S_{x}(\lambda) d \lambda \int_{\Lambda_{+}} S_{y}(\lambda) d \lambda}} .
$$

Expression (8) should be used for instance when we are interested in the comovement of the cyclical or seasonal components of two economic series: we have simply to select the frequency band of interest (for the cycle we could chose e.g. a period between 3 and 10 years) and to look at the dynamic correlation within this frequency band.

From (8) we see that, unfortunately, correlation within a frequency band cannot be consistently defined as a simple average of the values taken on by the dynamic correlation index within the band: a more complicated function, involving the spectra of both $x_{t}$ and $y_{t}$, is needed. Note that, in the particular case $\lambda_{1}=0, \lambda_{2}=\pi, \rho_{x y}\left(\Lambda_{+}\right)$reduces to the static correlation coefficient between $x_{t}$ and $y_{t}$. Hence we can recover static correlation starting from dynamic correlation. However, we have to 'weight' correlations at different frequencies by using information from the spectral densities $S_{x}(\lambda)$ and $S_{y}(\lambda)$.

More generally, correlation at different frequencies is not related to correlation at different leads and lags by a simple relation. Indicating with $\rho_{k}^{x}$ and $\rho_{k}^{y}$ the autocorrelations of $x_{t}$ and $y_{t}$ at lag $k$ and with $\rho_{k}^{x y}$ the cross-correlation at lag $k$, we have

$$
\rho_{x y}(\lambda)=\frac{\sum_{k=-\infty}^{\infty} e^{-i \lambda k}\left(\rho_{k}^{x y}+\rho_{-k}^{x y}\right) / 2}{\sqrt{\sum_{k=-\infty}^{\infty} e^{-i \lambda k} \rho_{k}^{x} \sum_{k=-\infty}^{\infty} e^{-i \lambda k} \rho_{k}^{y}}} .
$$


Conversely, by setting

$$
D_{x y}(\lambda)=\sqrt{\frac{S_{x}(\lambda) S_{y}(\lambda)}{\operatorname{var}\left(x_{t}\right) \operatorname{var}\left(y_{t}\right)}}
$$

we have

$$
\frac{\rho_{k}^{x y}+\rho_{-k}^{x y}}{2}=\int_{0}^{\pi} \rho_{x y}(\lambda) D_{x y}(\lambda) e^{i \lambda k} d \lambda .
$$

Hence, in order to obtain $\rho_{x y}(\lambda)$ we must use not only the cross-correlations but also the autocorrelations at different lags. In the other direction, we have to resort to the spectral densities; moreover, we cannot get the cross-correlations at different lags, but only the sum $\rho_{k}^{x y}+\rho_{-k}^{x y}$.

\subsection{Dynamic correlation and band-pass filtering}

As already observed, in the macroeconomic literature stylized facts on comovements are often presented in terms of correlations of HP filtered or band-pass filtered data. The idea is that if we want to see whether e.g. the cyclical components of the outputs of two nations are synchronized, we first have to extract the cycles from the outputs and then look at correlations.

This procedure is theoretically consistent with our measure, provided that we discard HP filtering and use instead the well-known two-sided filter

$$
A_{\Lambda}(L)=\frac{\lambda_{2}-\lambda_{1}}{\pi}+\sum_{k=1}^{\infty} \frac{\sin k \lambda_{2}-\sin k \lambda_{1}}{k \pi}\left(L^{k}+L^{-k}\right)
$$

where $L$ is the lag operator and $\Lambda=\Lambda_{+} \cup \Lambda_{-}$with $\Lambda_{+}=\left[\lambda_{1}, \lambda_{2}\right), \Lambda_{-}=\left[-\lambda_{2},-\lambda_{1}\right)$, and $0 \leq \lambda_{1}<\lambda_{2} \leq \pi .^{2}$

To see this, consider that

$$
A_{\Lambda}\left(e^{-i \lambda}\right)= \begin{cases}1 & \text { for } \lambda \in \Lambda \\ 0 & \text { otherwise }\end{cases}
$$

(see e.g. Sargent, 1987, Section 11.6, p. 259), so that the spectrum of $A_{\Lambda}(L) x_{t}$, which is equal to $\left|A_{\Lambda}\left(e^{-i \lambda}\right)\right|^{2} S_{x}(\lambda)$, is equal to $S_{x}(\lambda)$ for $\lambda \in \Lambda$ and is zero elsewhere. Hence the filter eliminates all the waves whose frequency is outside the relevant interval and leaves

\footnotetext{
${ }^{2}$ The above filter, unlike the HP filter, must be applied to stationary data, so that if we start from trended data we have first to take the differences.
} 
unchanged the amplitude of the waves within the interval. But this means that $A_{\Lambda}(L) x_{t}=$ $x_{\Lambda t}$. Similarly, $A_{\Lambda}(L) y_{t}=y_{\Lambda t}$. It follows that

$$
\operatorname{corr}\left(A_{\Lambda}(L) x_{t}, A_{\Lambda}(L) y_{t}\right)=\rho_{x y}\left(\Lambda_{+}\right)
$$

Clearly, the equivalence above is true for theoretical measures. In practice the two estimation procedures will give different results. When estimation is performed by prefiltering data, truncation of $A_{\Lambda}(L)$ is needed in order to apply it to a finite time series. On the other hand, estimation of $\rho_{x y}\left(\Lambda_{+}\right)$will raise issues related to the estimation of spectra and cross-spectra.

\subsection{Dynamic correlation and cointegration}

Dynamic correlation at frequency zero is related to the notion of 'stochastic cointegration'. We recall that the $I(1)$ processes $z_{t}$ and $w_{t}$ are 'stochastically cointegrated' if and only if there is a linear combination $z_{t}-a w_{t}$ which is trend-stationary, i.e. it is the sum of a stationary variable and a linear deterministic trend $b+c t$. Now let $z_{t}$ and $w_{t}$ be $I(1)$ and set $x_{t}=\Delta z_{t}, y_{t}=\Delta w_{t}$. Then $z_{t}$ and $w_{t}$ are stochastically cointegrated if and only if

$$
\rho_{x y}(0)^{2}=1
$$

i.e. dynamic correlation at frequency zero is either 1 or -1 .

This is easily seen by observing that $C_{x y}(0)=S_{x y}(0)$, so that if $\rho_{x y}(0)^{2}=1$ then $S_{x y}(0)^{2}-S_{x}(0) S_{y}(0)=0$, i.e. the determinant of the spectral-density matrix of the vector $\left(\begin{array}{cc}\Delta z_{t} & \Delta w_{t}\end{array}\right)^{\prime}$ vanishes at zero frequency.

\section{Cohesion and cross-cohesion}

Let us now consider a vector of $n \geq 2$ variables $x_{t}=\left(\begin{array}{lll}x_{1 t} & \cdots & x_{n t}\end{array}\right)^{\prime}$. Moreover, let us attach non-normalized positive weights $w=\left(\begin{array}{llll}w_{1} & \cdots & w_{n}\end{array}\right)^{\prime}$ to the variables in $x_{t}$. Our proposed measure for the internal cohesion of the variables in $x_{t}$ is motivated in the same manner as in the bivariate case and equals the weighted average of the dynamic correlations, as defined in (1), between all possible pairs of series. This weighted average will be called cohesion and equals

$$
\operatorname{coh}_{x}(\lambda)=\frac{\sum_{i \neq j} w_{i} w_{j} \rho_{x_{i} x_{j}}(\lambda)}{\sum_{i \neq j} w_{i} w_{j}}
$$


The measure of cohesion within the frequency band $\Lambda_{+}=\left[\lambda_{1}, \lambda_{2}\right]$ is analogously given by

$$
\operatorname{coh}_{x}\left(\Lambda_{+}\right)=\frac{\sum_{i \neq j} w_{i} w_{j} \rho_{x_{i} x_{j}}\left(\Lambda_{+}\right)}{\sum_{i \neq j} w_{i} w_{j}} .
$$

A very simple choice for the weights is $w_{i}=1$ for all $i$. While equal weights may work well in many cases, they appear unsuited when we deal with sectors or regions with very different importance. For instance, if we want to measure the cohesion of the per-capita incomes of the European countries, it is reasonable to give Germany a greater weight than Luxembourg. A natural choice for $w_{i}$ in this case would be the level of income or population of country $i$ at some $t$.

We exclude the diagonal terms in the weighted average (10) for two reasons. First, it seems reasonable to require that, if the entries in $x_{t}$ are pairwise uncorrelated at all leads and lags, cohesion is zero at all frequencies. This property hold with the above definition, whereas it would be violated when including the diagonal terms. Second, the inclusion of the diagonal terms would render cohesion dependent of $n$. As an example, assume $\rho_{x_{i} x_{j}}(\lambda)=\rho$ for $i \neq j$ and at all frequencies $\lambda$, and set for simplicity $w_{i}=1$ for all $i$. According to definition (10) we get $\operatorname{coh}_{x}(\lambda)=\rho$, while the inclusion of the diagonal terms would give $[\rho(n-1)+1] / n$, so that a group with two uncorrelated variables would have cohesion 0.5 while a group with ten pairwise uncorrelated variables would have cohesion 0.1.

Clearly $\left|\operatorname{coh}_{x}(\lambda)\right| \leq 1$ and $\operatorname{coh}_{x}(\lambda)=1$ if and only if all of the variables in $x_{t}$ comove perfectly at frequency $\lambda$. In particular, $\operatorname{coh}_{\Delta z}(0)=1$ if and only if the variables in $z_{t}$ are pairwise cointegrated with cointegrating vector $\left(\begin{array}{ll}1-1 \\ -\end{array}\right)$ i.e. they have a common trend representation and equal long run responses to the permanent shock. Moreover, $\operatorname{coh}_{x}(\lambda)=1$ at each frequency if and only if all of the $x_{i t}$ 's are identical upto a linear transformation.

The lower bound of the cohesion index is -1 for $n=2$, since, if $n=2$, the cohesion of $x_{t}$ coincides with the dynamic correlation index of $x_{1 t}$ and $x_{2 t}$ (in dependently of $w$ ). For $n>2$ the lower bound is greater, since of course we cannot have perfect pairwise negative correlation within a group of three variables or more. To illustrate this point, note that (10) can be rewritten as $\operatorname{coh}_{x}(\lambda)=\left(w^{\prime} R_{\lambda} w-w^{\prime} w\right) / \sum_{i \neq j} w_{i} w_{j}$, where $R_{\lambda}$ is like a correlation matrix, but now containing all pairwise dynamic correlations at frequency $\lambda$. This expression cannot be less than $-w^{\prime} w / \sum_{i \neq j} w_{i} w_{j}$, which value depends on the particular choice of the weights. An example is the case of equal weights, with the minimum $1 /(n-1)$ tending to zero as $n$ gets larger. 
Note that, while pairwise correlation implies zero cohesion, the converse is not true: when observing a small cohesion index we cannot distinguish whether it originates from small pairwise comovements or large negative and positive covariances canceling out each others. This problem could be avoided by using the alternative measure

$$
\operatorname{coh}_{x}^{*}=\frac{\sum_{i \neq j} w_{i} w_{j}\left|\rho_{x_{i} x_{j}}\right|}{\sum_{i \neq j} w_{i} w_{j}}
$$

On the other hand, $\operatorname{coh}_{x}^{*}$ has the disadvantage that it does not distinguish between negative and positive comovements. If we want to retain both informations we can do this by comparing coh and $\operatorname{coh}^{*}$.

The cohesion index can be easily generalized to an index measuring the cross-cohesion between the $n$-vector $x_{t}$ and the $m$-vector $y_{t}$. For the sake of simplicity, let us assume for the moment that $x_{t}$ and $y_{t}$ have no common elements. Let us specify two vectors of weights $w_{x}$ and $w_{y}$. Cross-cohesion of $x_{t}$ and $y_{t}$ at frequency $\lambda$ is then given by

$$
\operatorname{coh}_{x y}(\lambda)=\frac{\sum_{i=1}^{n} \sum_{j=1}^{m} w_{x_{i}} w_{y_{j}} \rho_{x_{i} y_{j}}(\lambda)}{\sum_{i=1}^{n} \sum_{j=1}^{m} w_{x_{i}} w_{y_{j}}}
$$

Note that if both $x_{t}$ and $y_{t}$ are scalars, cross-cohesion reduces to dynamic correlation.

In some cases it could be interesting to evaluate the cross-cohesion of two overlapping sets of variables. In this case we should eliminate from the weighted average the self-comovements of the variables in the intersection, as we have done in defining the cohesion index (10). Hence the above definition of cross-cohesion should be generalized in the following way. For notational simplicity, let us reorder the variables in $x_{t}$ and $y_{t}$ in such a way that, if $x_{t}$ and $y_{t}$ have $k$ variables in common, the latter variables occur in the first $k$ places. The generalized index of cross-cohesion is then

$$
\frac{\sum_{i=1}^{n} \sum_{j=1}^{m} w_{x_{i}} w_{y_{j}} \rho_{x_{i} y_{j}}(\lambda)-\sum_{i=1}^{k} w_{x_{i}} w_{y_{j}}}{\sum_{i=1}^{n} \sum_{j=1}^{m} w_{x_{i}} w_{y_{j}}-\sum_{i=1}^{k} w_{x_{i}} w_{y_{i}}} .
$$

According to this definition, the cross-cohesion of $x_{t}$ with $x_{t}$ is equal to the cohesion of $x_{t}$.

\section{Empirical application: business cycles in Europe and the US}

In this Section we evaluate the issue of comovement of output fluctuations between European countries and US states and regions. This problem has been analyzed by many authors; a 
comprehensive review of the literature is provided by Clark and Shin (1999). On the basis of our measure of cohesion we are able to bring attention to some features of comovement that have been overlooked in previous studies.

We analyze two panels of data: the personal income of the 51 US states and the GDP of seventeen West-European nations. Data are yearly, from 1962 to 1997. A European core composed by the 11 EMU countries and the 8 BEA regions are also analyzed separately. We look at US regions, in addition to states, since the size the former is larger and comparable with that of the largest European nations. The series are taken in log-difference to render them stationary. (More information on the data is found in Appendix I).

Let us articulate our analysis around a few questions.

- Are national cycles within Europe more synchronized than regional and state cycles within the US?

Figure 2 shows cohesion of US regions, US states, EMU countries and European countries (including EMU) ${ }^{3}$. As expected, European countries are less cohesive than EMU countries, which in turn are less cohesive than US states at all frequencies. Finally, US regions are more cohesive than US states, since, by aggregating the states, the idiosyncratic sources of variations are reduced. This corresponds to the consensual view.

However, while at business cycle frequencies (around 1.5, corresponding to a period of about 4 years) the difference between Europe and US is large, at low frequencies the difference is much smaller. This asymmetry between long-run and short-run has not been emphasized in the literature. Figure 3 reports the curves for US states and EMU countries along with $2 \sigma$ bootstrap confidence bands (see Appendix II for details). The bands do not overlap at cyclical frequencies, whereas there is a very large overlapping in the long-run. The same result holds when comparing the larger set of the European countries with US regions (the confidence bands are not reported here). We conclude that, as far as synchronization of long cycles is concerned, the difference between Europe and the US is small and non-significant, while the opposite holds for the typical business cycle frequencies.

- Are European nations more correlated with other European nations than with US states?

A first answer is given by Figure 4, which reports again within cohesion of US states and

\footnotetext{
${ }^{3}$ To estimate the spectra intervening in the definition of dynamic correlation, periodograms smoothed with a Bartlett window with lag window size 6 were used.
} 


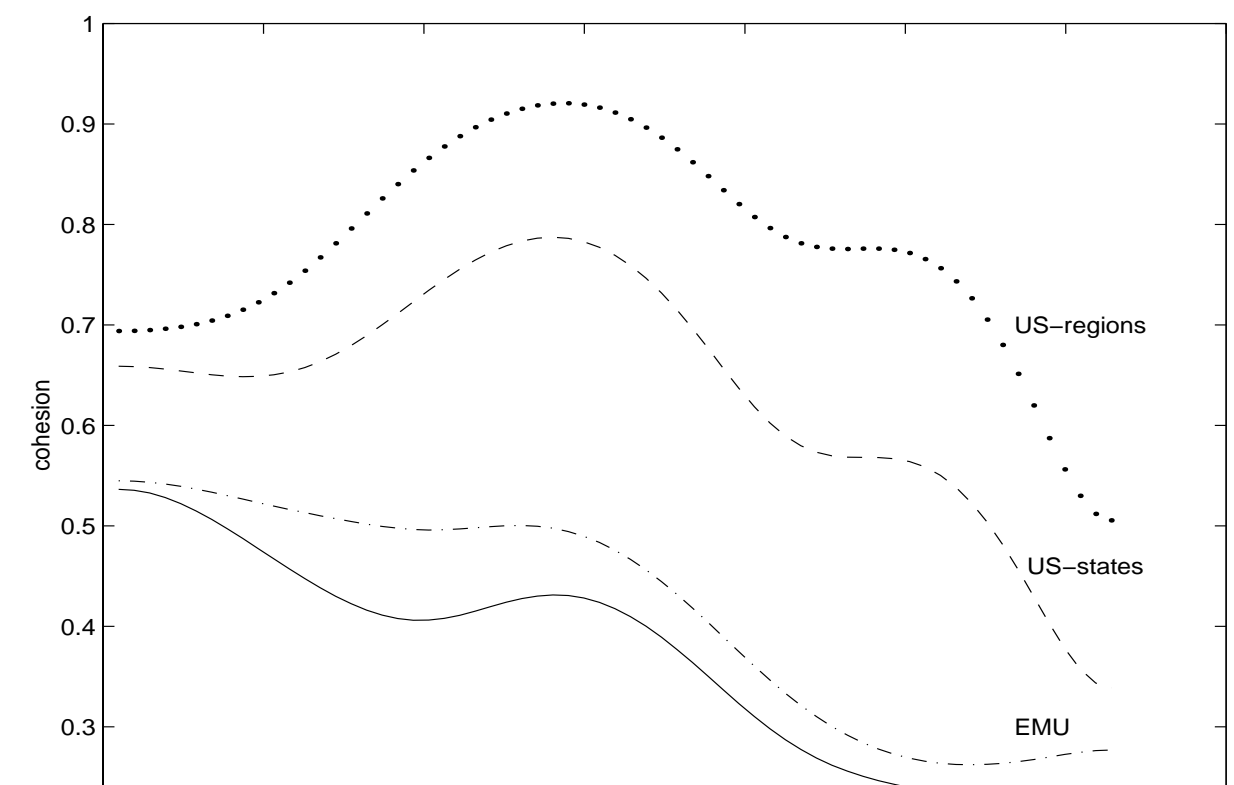

Figure 2: Cohesion of the 51 US -states, the 8 US-regions, the grouteprogf 17 West-European Countries, and the 11 EMU-members.

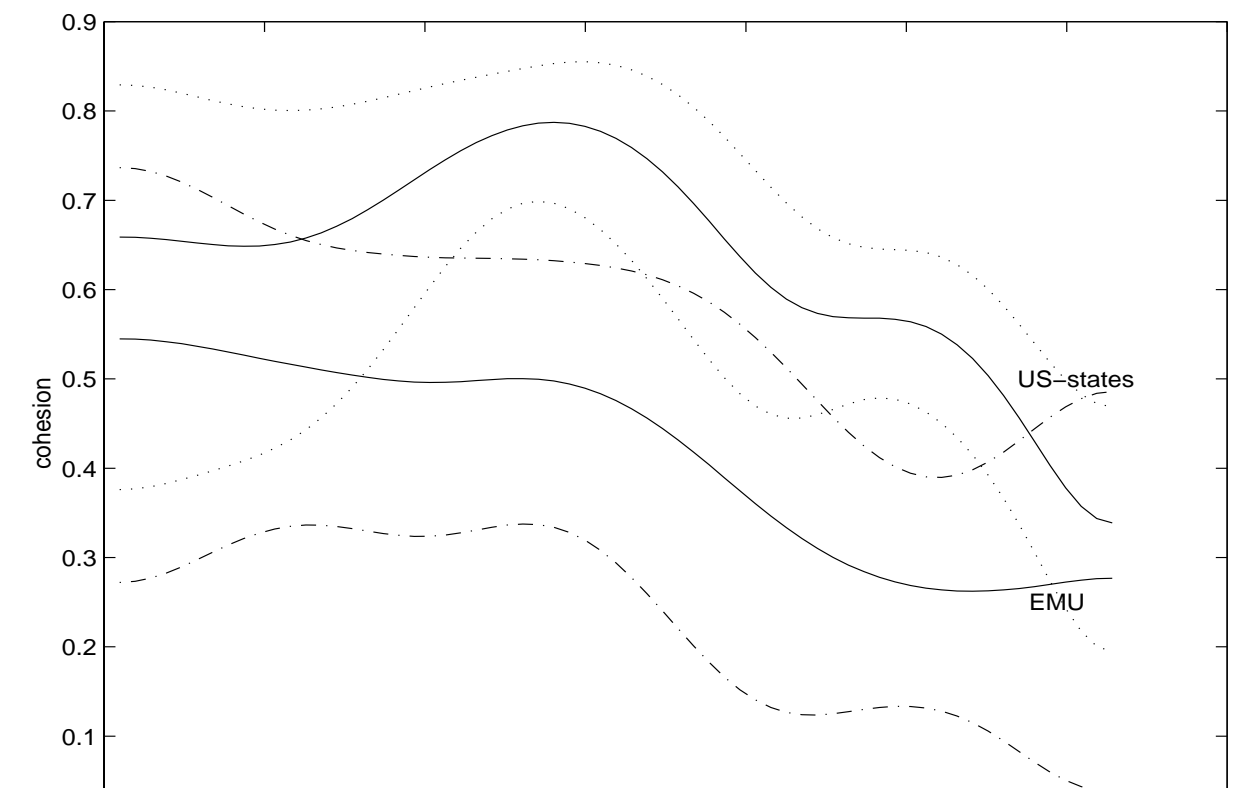

Figure 3: Cohgsion for US and EMU-members. Confidence bounds for US are indicated by ‥ and for EMU by -. 


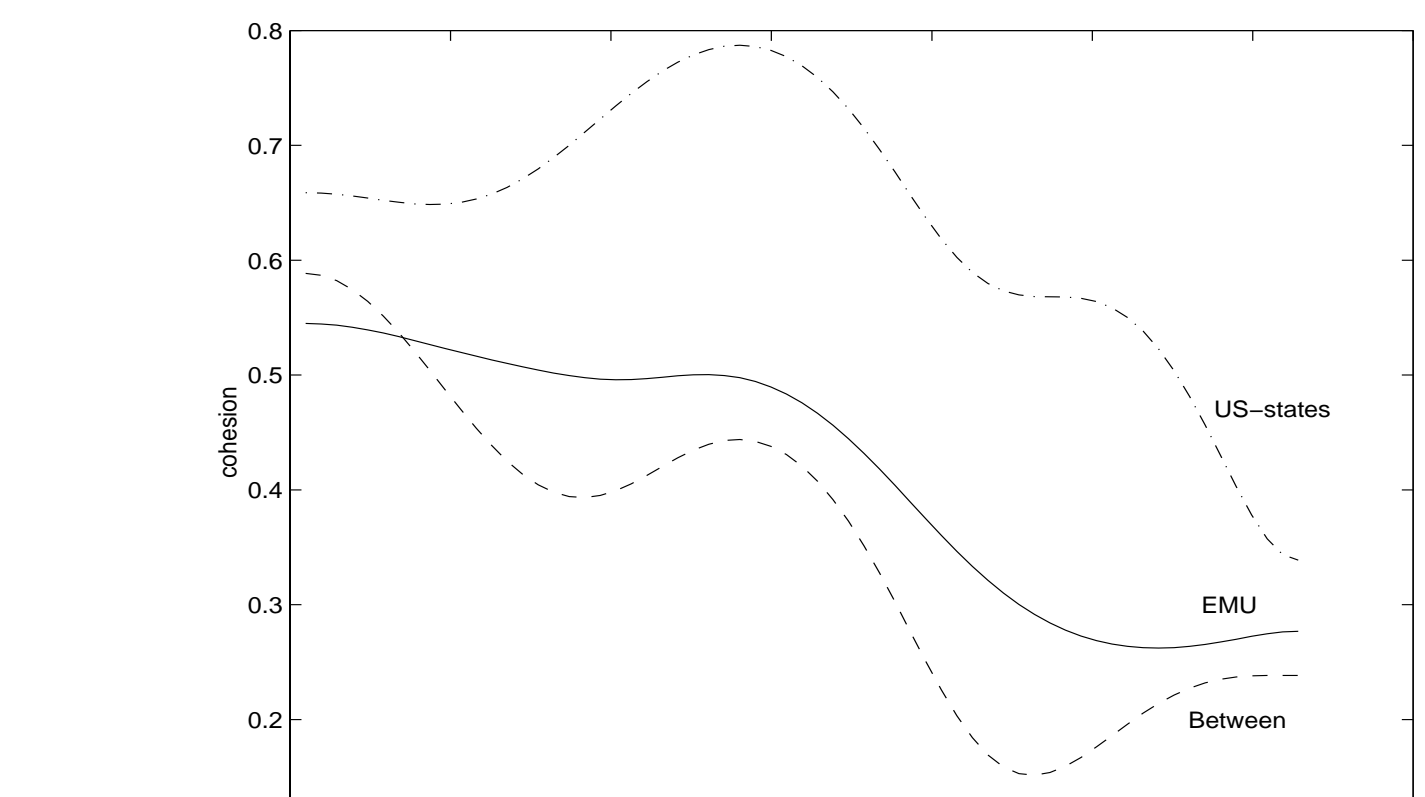

Figure 4: Within and Cross-Cohesion of the group of $\begin{gathered}51 \\ 0.5\end{gathered} \frac{1}{2}$ US-states, $_{3}$ and the group of 11 EMU-members.

EMU countries, along with cross-cohesion between US and Europe. We see that within cohesion of EMU countries is larger than cross-cohesion, but again differences disappear in the long-run.

Another way to look at the problem is to use cohesion as a measure of distance in computing clusters. Our purpose here is to group the $50 \mathrm{US} \mathrm{states}^{4}$ and the 17 European countries (cfr. Appendix I) according to the way that their national incomes comove together in the short run and the long run.

We proceed as follows. We first compute dynamic correlations $\rho_{i j}\left(\Lambda_{+}\right)$between all pairs of countries/states for a given frequency band $\Lambda_{+} \cdot{ }^{5}$ Then the 67 by 67 matrix of (positive) "dissimilarities" $D\left(\Lambda_{+}\right)$is computed. This matrix has elements

$$
D_{i j}\left(\Lambda_{+}\right)=1-\rho_{i j}\left(\Lambda_{+}\right)
$$

Countries which strongly comove within the given frequency band, have small "dissimilarities".

\footnotetext{
${ }^{4}$ Alaska was excluded from the cluster analysis since it appeared to be, not surprisingly, a huge outlier.

${ }^{5}$ In practice, the integral appearing in definition (8) of $\rho_{i j}\left(\Lambda_{+}\right)$was computed by numerical integration.
} 
To distinguish between short-run and long-run dynamics, we perform calculations for two different frequency ranges, corresponding to cycles of period of eight years or longer (longrun dynamics) and cycles of period less than eight years, representing short-run fluctuations and business cycle dynamics.

Once the dissimilarity matrix has been computed, standard clustering techniques can be applied. We chose to apply the metrical multidimensional scaling ${ }^{6}$ technique (Cox and Cox, 1994) which represents the 67 countries and states in a 2 dimensional plane. The Euclidean distances between the points in the plane are now supposed to mimic the dissimilarities computed by (13). Countries which have big dissimilarities, have representations in the plane which are far away from each other. Looking at the objects in the two-dimensional plane, makes it possible to detect possible clusters and outliers.

In Figure 5 we illustrate results of the application of multidimensional scaling based on the cohesion indices for long run comovements, and for business cycle and short run comovements. European countries are labeled in the standard way while US states are labeled according to whether they belong to region $1,2, \cdots, 8$. The axes in the figure cannot be interpreted, only the relative distances between pairs of states are important.

The result of Figure 4 are confirmed: once again, dynamics matter. In the short-run, European countries are clustered on one side of the box and US regions on the other side with UK and Ireland being the closest to US states (this confirms results found in Forni and Reichlin, 1999). In the long-run, however, no clear pattern emerges and units belonging to the US are mixed together with units belonging to Europe. In the long-run, US states are as correlated with each other than as with European nations, probably reflecting the effect of a common persistent shock.

\section{- Does border matter?}

The question of whether business cycles of states or countries which are close from a geographical point are more correlated is of crucial interest to understand the nature of the propagation mechanism of economic shocks. A robust finding in the literature is that geography matters for trade and recently it has been found that it also matters for output correlation (Clark and van Wincoop, 1999). Our purpose here is to evaluate the latter claim for our data set taking into account dynamics. To this purpose, we compute, for each state

\footnotetext{
${ }^{6}$ The procedure cmdscale of the statistical software package Splus was used for this.
} 

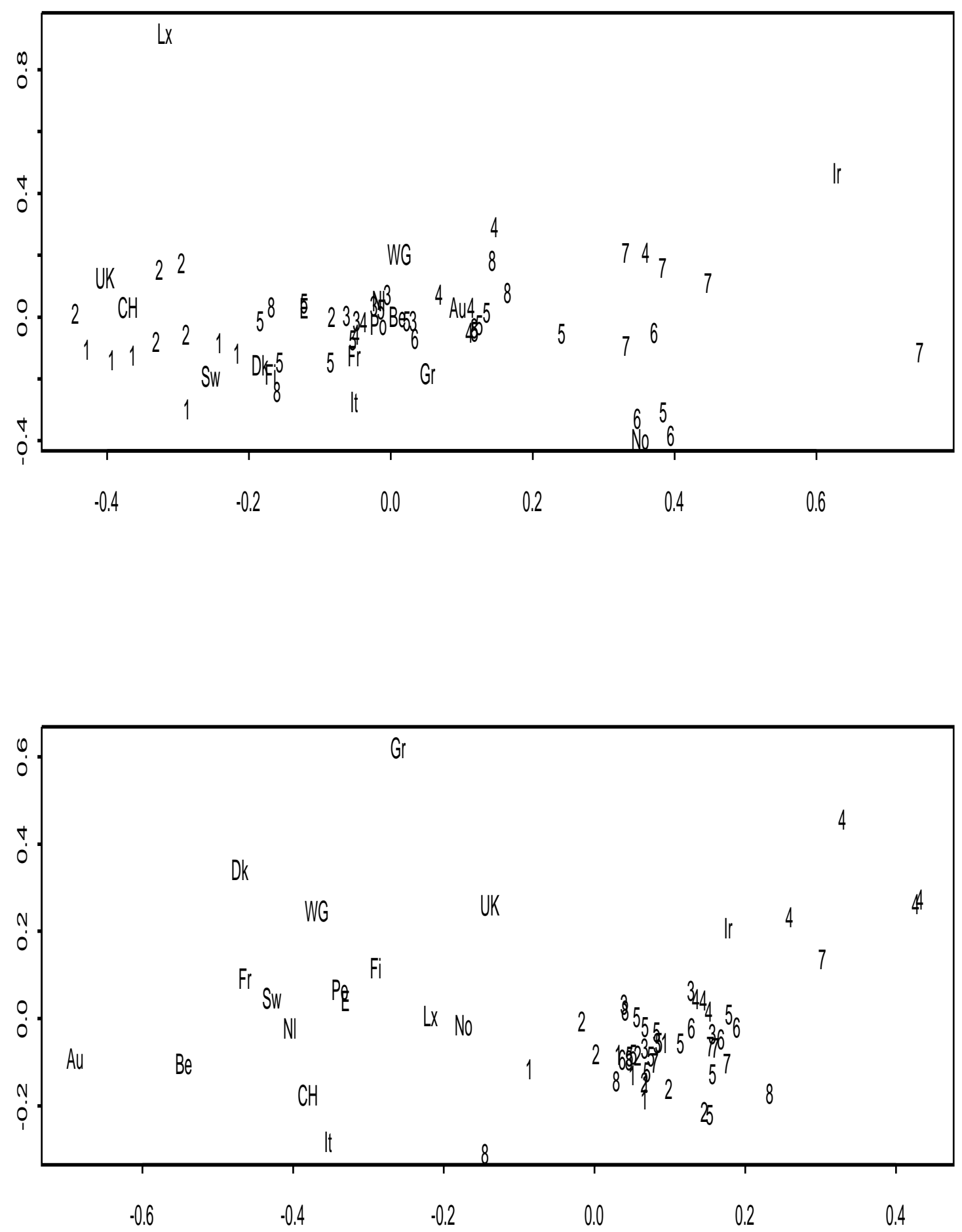

Figure 5: Representation of the 50 US-states (Alaska is excluded) and the 17 West-European countries in a plane according to their long run comovements (upper figure) and short run comovement (lower figure). The US-states are labeled by the regions to which they belong. 
(and country), the ratio between average cohesion with neighbors states (and countries) and average cohesion with all states (and countries). This can be taken as a measure of the extent to which "border matters".

For each US state and European nation we compute the following measure

$$
\operatorname{BM}_{i}\left(\Lambda_{+}\right)=\frac{\text { average }_{j \in N_{i}} \rho_{i j}\left(\Lambda_{+}\right)}{\text {average }_{j \neq i} \rho_{i j}\left(\Lambda_{+}\right)},
$$

where $N_{i}$ is the set of indices of all neighbor states of state $i$ and $\rho_{i j}\left(\Lambda_{+}\right)$is the dynamic correlation between states $i$ and $j$ at the selected frequency band $\Lambda_{+}$.

Figure 6 show results for US states. The ratio (14) has been computed for every state (with the exception of Alaska and Hawaii, which have no neighbors) and for long run frequencies $\left(\Lambda_{+}=[0, \pi / 4]\right)$ and short run frequencies $\left(\Lambda_{+}=[\pi / 4, \pi]\right)$, as was done in the clustering example. The maps tell us that in the short-run most states are as correlated with the neighbors as with all other states, since their $\mathrm{BM}_{i}$ is close to 1 . This is not surprising, given the large cohesion between US states that we had found at business cycle frequencies (Figure 2). The result confirms, in a different way, that there is a lot of common dynamics at business cycle frequencies. In the long-run, however, there are states which comove more strongly with the neighbors (this does not imply that those states comove strongly with their neighbors, it just says that they are comoving more strongly with their neighbors than with the other states). Notice that, in general, the ratios are above one, indicating that border matters. ${ }^{7}$ How much border matters, however, depends in the first place on the size of the state, which is quite natural .

Table 1 illustrates results for Europe. These results should be interpreted with caution since, due to the small number of neighboring nations, p-values from the non-parametric Wilcoxon-rank test indicate that the difference of correlation with neighbors and correlation with all nations is generally statistically not significant. Qualitatively, we can observe, however, that border in Europe matters a little (the ratios are generally larger than one), but less than in the US. Moreover, no difference in patterns emerges between short-run and long-run ratios. The weak effect of the border for nations contrasts with what found at the regional level (Quah, 1996). An interpretation, also suggested by what found by Forni and

\footnotetext{
${ }^{7}$ The non-parametric Wilcoxon-rank test indicated that average long-run comovements with neighbors are significantly different (at the $5 \%$ level) from the average long-run comovements with all states for more than $60 \%$ of the states. Results are available upon request.
} 
long run

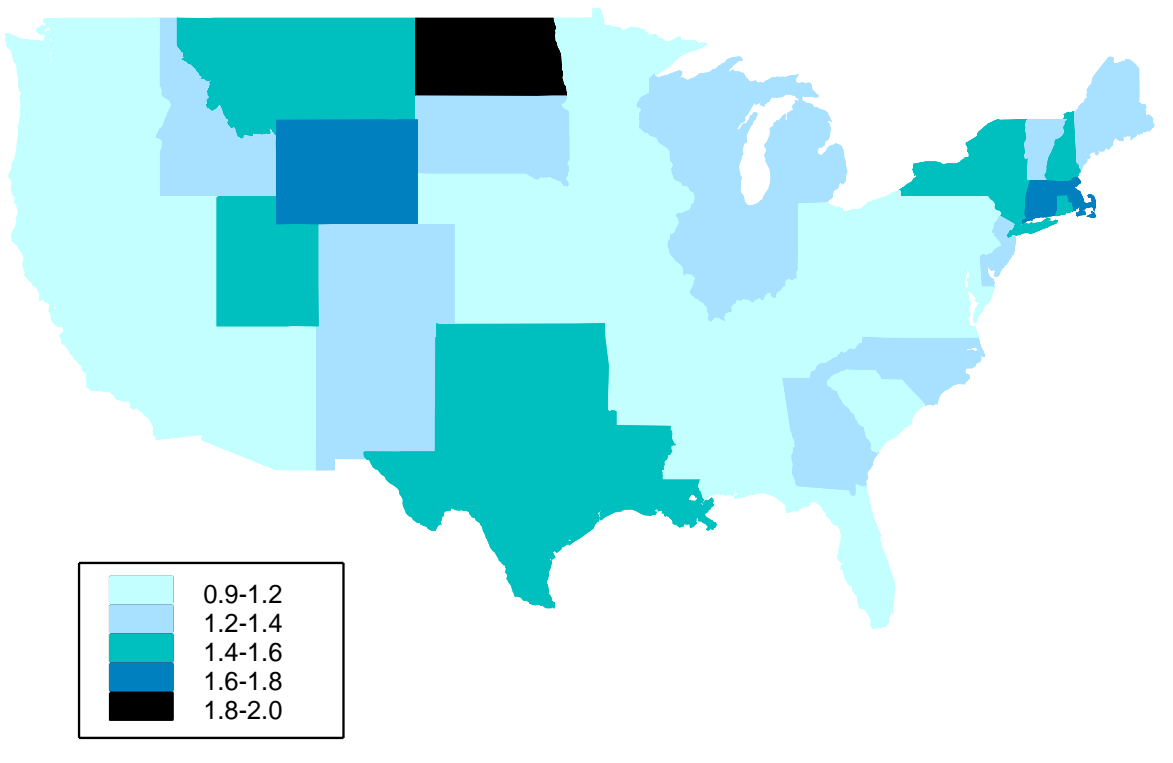

short run

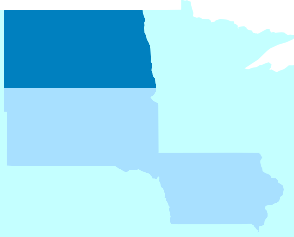

$0.9-1.2$

$1.2-1.4$

1.4-1.6

Figure 6: The 50 ate.8-2.8laska and Hawii are excluded) colored according to the value of their border-correlation-measure BMi for the long run (upper figure) and the short run (lower figure). 


\begin{tabular}{|r|ll|ll|c|}
\hline Country & \multicolumn{2}{|l|}{ Long run BM } & \multicolumn{2}{|l|}{ Short run BM } & Neighbors \\
\hline $\mathrm{Au}$ & 1.15 & $(0.93)$ & 1.31 & $(0.05)$ & 3 \\
$\mathrm{Be}$ & 1.00 & $(0.50)$ & 1.16 & $(0.05)$ & 4 \\
$\mathrm{Dk}$ & 0.87 & - & 1.67 & - & 1 \\
$\mathrm{Fi}$ & 1.07 & $(0.76)$ & 0.99 & $(0.91)$ & 2 \\
$\mathrm{Fr}$ & 1.00 & $(0.73)$ & 1.17 & $(0.01)$ & 6 \\
$\mathrm{WG}$ & 1.17 & $(0.13)$ & 1.22 & $(0.00)$ & 7 \\
$\mathrm{It}$ & 1.24 & $(0.16)$ & 1.26 & $(0.07)$ & 3 \\
$\mathrm{Lx}$ & 2.14 & $(0.21)$ & 1.33 & $(0.05)$ & 3 \\
$\mathrm{Nl}$ & 1.26 & $(0.15)$ & 1.35 & $(0.01)$ & 2 \\
$\mathrm{Po}$ & 1.25 & - & 1.15 & - & 1 \\
$\mathrm{E}$ & 1.26 & $(0.15)$ & 1.33 & $(0.23)$ & 2 \\
$\mathrm{Sw}$ & 0.87 & $(0.76)$ & 1.15 & $(0.63)$ & 2 \\
$\mathrm{CH}$ & 1.19 & $(0.26)$ & 1.23 & $(0.05)$ & 4 \\
$\mathrm{No}$ & 1.60 & $(0.63)$ & 0.45 & $(0.41)$ & 2 \\
\hline
\end{tabular}

Table 1: Borders Measure $\mathrm{BM}_{i}$ for the long run and the short run computed for 14 European countries. P-values are given between parenthesis and the number of neighbors is in the last column.

Reichlin (1999), is that European nations contain clusters of regions with different degree of synchronization and that geographical effects are not defined by political boundaries.

\section{Summary and conclusion}

This paper has proposed a measure of dynamic comovement between (possibly many) time series and named it cohesion. The measure is defined in the frequency domain and it is appropriate for costationary processes or, as it is often the case with macroeconomic time series, processes which are costationary in first differences. In the bivariate case, the measure is shown to correspond to dynamic correlation and it is related, but not equal, to the well known quantities "coherence" and "coherency". The multivariate measure is the corresponding weighted average. We show that cohesion relates in a simple way to cointegration.

Cohesion is useful to study problems of business cycle synchronization, to investigate 
short-run and long-run dynamic properties of multiple time series, to identify dynamic clusters. It is also the appropriate tool to establish stylized facts on comovements of macroeconomic variables at specific frequency bands of interest.

Our empirical analysis on cohesion of output fluctuations within the US, within Europe and between US and Europe illustrates the importance of the dynamic decomposition of comovements. Results show that, in the long run (cycles longer than four years), cohesion of output fluctuations within the US is not significantly different than cohesion within Europe and that US states are as correlated with each other than as with European nations, probably reflecting the effect of a common persistent shock. In addition, we provide results on the geographical structure of cohesion. We show that, in general, states (or nations) are more strongly cohesive with neighbors states than with other states (or nations). In the US, this effect is stronger in the long-run than in the short-run.

\section{REFERENCES}

Ahn, S.K. and Reinsel, G.C. (1988) "Nested reduced-rank autoregressive models for multiple time series" Journal of the American Statistical Association, 83, 849-856.

Berkowitz, J. and Kilian, L., (1996). "Recent Developments in Bootstrapping Time Series", Finance and Economics Discussion Series, Federal reserve Board, Washington, D.C., 1996-45

Berkowitz, J. and Diebold, F.X., (1998), "Bootstrapping Multivariate Spectra,", Review of Economics and Statistics, , 80, 664-66.

Clark, T. and Shin, K. (1999), "The sources of fluctuations within and across countries", in G.D.Hess and E. van Wincoop, eds., Intranational Macroecoonomics, Cambridge University Press, forthcoming.

Clark, T. and van Wincoop, E. (1999) "Borders and business cycles", paper presented at the conference "Lessons from intranational economics for international economics, June 11-12 at Studienzentrum Gerzensee, Switzerland.

Cox, T. F., and Cox, A.A. (1994), Multidimensional scaling, London: Chapman \& Hall. 
Engle, R.F. and Granger, C.W. J. (1987), "Cointegration and error correction: representation, estimation and testing", Econometrica, 55, 251-276.

Engle, R.F. and Kozicki, S. (1993) "Testing for common features", Journal of Business 8 Economic Statistics, vol. 11, n. 4.

Forni, M. and Reichlin, L., (1996) "Dynamic common factors in large cross-sections", Empirical Economics 21, pp. 27-42.

— nomic Review, forthcoming.

— dynamics", July, Review of Economic Studies.

Gourieroux, C., and Peaucelle, I. (1992), "Series codépendantes: Application a l'hypothèse de parité du pouvoir d'achat", l'Actualité Economique 68, pp 283-304.

Lilien, D. M. (1982) "Sectoral shifts and cyclical unemployment", Journal of Political Economy, August, pp. 777-93.

Quah, D. (1993) "Comment", Journal of Business 8 Economics and Statistics, vol. 11, No. 4.

Quah, D. (1996) "Regional convergence clusters across Europe", European Economic Review, vol. 40 , no3-5.

Sargent, T. (1987), Macroeconomic Theory, 2nd edition, Academic Press, Orlando.

Vahid, F. and Engle, R.F. (1993), "Common trends and common cycles", Journal of Applied Econometrics, 8, 341-60. 


\section{APPENDIX 1:}

Data sources and data treatment

A. US states personal income: The data are "Personal income (USD)" from REIS, database provided by the Bureau of Economic Analysis, Economics and Statistics Administration of the US department of Commerce. The data were deflated by a current price index (annual, all urban consumers). The BEA-regions are labeled as New England (1), Mideast (2), Great Lakes (3), Plains (4), Southeast (5), Southwest (6), Rocky Mountain (7), and the Far West (8).

B. European nations GDP: The data are GDP at 1990 market prices deflated by the GDP deflator (Mrd USD 1990). The source is Eurostat. Countries considered are Austria (Au), Belgium (Be), West Germany (WG), Spain (E), France (Fr), Ireland (Ir), Italy (It), Luxembourg (Lx), Netherlands (Nl), Portugal (Po), Finland (Fi) which form the 11 EMU-members. Furthermore, 6 other countries were added to constitute the set of 17 "West-European" countries: Switzerland (Ch), Norway (No), Sweden (Sw), Greece (Gr), Denmark (Dk).

\section{APPENDIX 2:}

Bootstrapping technique

Confidence limits around the estimated cohesion coefficients were computed using bootstrap techniques. An overview of methods for bootstrapping time series is given in Berkowitz and Kilian (1996). We applied a standard block bootstrap for the cohesion of real personal income within US and GDP within the EMU-countries, respectively (cfr. Section 4). The number of replicates was 200 and the length of the blocks was chosen to be equal to 7 , large enough to retain the cyclical information in the series.

The distribution of the bootstrap replicates was relatively close to a normal one. To get even more close to a normal distribution, the Fisher z-transformation has been applied to the simulated dynamic correlations. The Fisher z-transformations tranfers correlations $\rho$ into $\frac{1}{2} \ln ((1+\rho) /(1-\rho))$, where the latter may take on values on the whole real line. Confidence limits were then computed on the transformed data by the $2 \sigma$-rule, and retransformed, by applying the inverse of the Fisher z-transformation, into a confidence interval for the actual cohesion $\operatorname{Coh}(\lambda)$. 
Since cohesion is defined in the frequency domain, it is very appealing to use the spectral approach of Berkowitz and Diebold (1998). By bootstrapping the (multivariate) spectrum instead of the time series itself, we can save computation time, since the spectrum does not need to be recomputed for each bootstrap replicate. Preliminary experiments in this direction were not so successful, probably due to the fact that the time span in the treated examples is rather small. Research on this topic is ongoing. 